\title{
International Journal of Anesthesia and Relaxation
}

Exploring Scientific Community

Case Report

Open Access

Systolic Anterior Motion of the Mitral Valve after Aortic Valve Replacement for Aortic Stenosis diagnosed by Transesophageal Echocardiography

John F. Zaki*, Stephanie F. Tran, Travis Markham, Warren Choi, Rodolfo E. Perez, Timothy R. Pawelek, Ovidiu Moise, Shaina Sheppard, Ali Shahen, Shao F. Zhou, Sreelatha Panthayi, Marty N. Giesecke, Roy Sheinbaum.

Department of Anesthesiology, University of Texas Health Science Center at Houston. 6431 Fannin Street, MSB 5.020 Houston, Texas. ${ }^{*}$ Corresponding Author: John F. Zaki, MD Department of Anesthesiology, University of Texas Health Science Center at Houston 6431 Fannin Street, MSB 5.020 Houston, TX 77030 Tel: (832) 541-5835 Email: john.f.zaki@uth.tmc.edu

Citation: John F. Zaki, et al (2017) Systolic Anterior Motion of the Mitral Valve after Aortic Valve Replacement for Aortic Stenosis diagnosed by Transesophageal Echocardiography Int J Ane \& Rel. 1:1, 16-18

Copyright: (C2017 John F. Zaki, et al This is an open-access article distributed under the terms of the Creative Commons Attribution License, which permits unrestricted use, distribution, and reproduction in any medium, provided the original author and source are credited.

Received September 20,2017; Accepted September 27, 2017; Published September 29, 2017.

Keywords: Aortic stenosis, aortic valve replacement, systolic anterior motion, transesophageal echo

\section{Introduction}

Systolic anterior motion of the mitral valve (SAM) is a paradoxical motion of the anterior, and occasionally posterior, mitral valve leaflet towards the left ventricular outflow tract (LVOT) during systole. Although SAM is well-reported in cases of hypertrophic cardiomyopathy, there are relatively fewer cases that describe severe SAM causing significant hemodynamic instability in association with aortic valve replacement (AVR) for aortic valve stenosis (1-3). With the likely underreported instances and consequent limited guidelines to non-surgical approach to treatment, the authors believe that our case adds valuable insight to the identification and treatment of an under-recognized cause of hemodynamic instability after AVR (1-3). We present a case of severe SAM developing intraoperatively after aortic valve replacement (AVR) for aortic valve stenosis, and discuss possible physiologic causes, importance of early identification, and initial treatment of this phenomenon.

Case Report:

A 62 -year-old $163 \mathrm{~cm}, 65 \mathrm{~kg}$ female with a prior past medical history consisting of hypertension and hyperlipidemia was recently diagnosed with severe aortic valve stenosis following increased exercise intolerance, chest pain, and multiple episodes of syncope. Her preoperative transthoracic echocardiogram (TTE) showed a preserved ejection fraction of $60 \%$, aortic valve area (AVA) 0.5 $\mathrm{cm}^{\wedge} 2$, mean gradient of $58.5 \mathrm{mmHg}$, and an LVOT of $1.7 \mathrm{~cm}$. She presented to our service for an elective minimally invasive AVR via a right anterior thoracotomy approach. Her home medications included aspirin $(81 \mathrm{mg})$, and Cardizem $(240 \mathrm{mg})$.

General anesthesia was induced with midazolam (3 mg), fentanyl $(50 \mu \mathrm{g})$, and propofol $(30 \mathrm{mg})$, while transient hypertension was treated with esmolol $(30 \mathrm{mg})$. Maintenance of general anesthesia was achieved by a combination of fentanyl, midazolam, and sevo- flurane. Preoperative diagnosis were confirmed with intraoperative transesophageal echocardiography, which showed a heavily calcified tri-leaflet aortic valve. AVA was determined to be 0.45 $\mathrm{cm}^{\wedge} 2$, peak velocity of $4.2 \mathrm{~m} / \mathrm{s}$, mean gradient of $62 \mathrm{mmHg}$, and LVOT diameter of $1.7 \mathrm{~cm}$ with preserved left ventricular systolic function. The pre-cardio pulmonary bypass (CBP) period was uneventful and the aortic valve was replaced using a \#23 St. Jude Trifecta Tissue aortic valve (St. Jude Medical, St. Paul, MN). The valve was secured using COR-KNOT and the aorta was then closed with a 4-0 Prolene suture.

Prior to weaning from CBP, no inotropic support was necessary as the patient was relatively healthy with the aforementioned TEE findings. However, during the weaning process, hemodynamic instability was unexpectedly encountered. SAM and subsequent left ventricular outlet obstruction, which had not been evident priot to AVR was subsequently identified on TEE. The remainder of the exam showed a normal functioning bio-prosthetic valve with an AVA of $3.2 \mathrm{~cm}^{\wedge} 2$. The pressure gradient across the LVOT was measured to be $65 \mathrm{mmHg}$ and the LVOT diameter was measured to be about $1.2 \mathrm{~cm}$ during observed SAM. Additionally, the severe mitral regurgitation with SAM was clearly identifiable (Fig 1, Video 1-2). M-mode confirmed the diagnosis with coarse fluttering of the aortic valve leaflets. Unstable hemodynamics persisted as the patient became tachycardic with heart rate into the $120 \mathrm{~s}$ and hypotensive with systolic blood pressure readings less than 80 and a mean arterial pressure in the 50s. Full CPB was continued while the decision was made to attempt to medically manage the situation with volume loading and with beta blockade. An additional 50 $\mathrm{mg}$ of esmolol was given to temporize the increased contractility that was exacerbating the SAM while aggressive volume loading was initiated simultaneously to increase the left ventricular end 
diastolic volume. This medical management during continued bypass for LVOT obstruction led to stabilization of her hemodynamics and improvement to a minimal amount of SAM (Gradient?). Consequently, successful weaning from CPB was accomplished.

The patient was transferred immediately to the cardiovascular intensive care unit and extubated on the first post-operative day.
She was started on beta-blocker therapy with metoprolol during her post-operative course. Her stay was complicated by new onset atrial fibrillation with rapid ventricular response which eventually resolved after a course of amiodarone, continued beta-blocker therapy, and close attention to her fluid status. She was discharged to home on post-operative day 10.

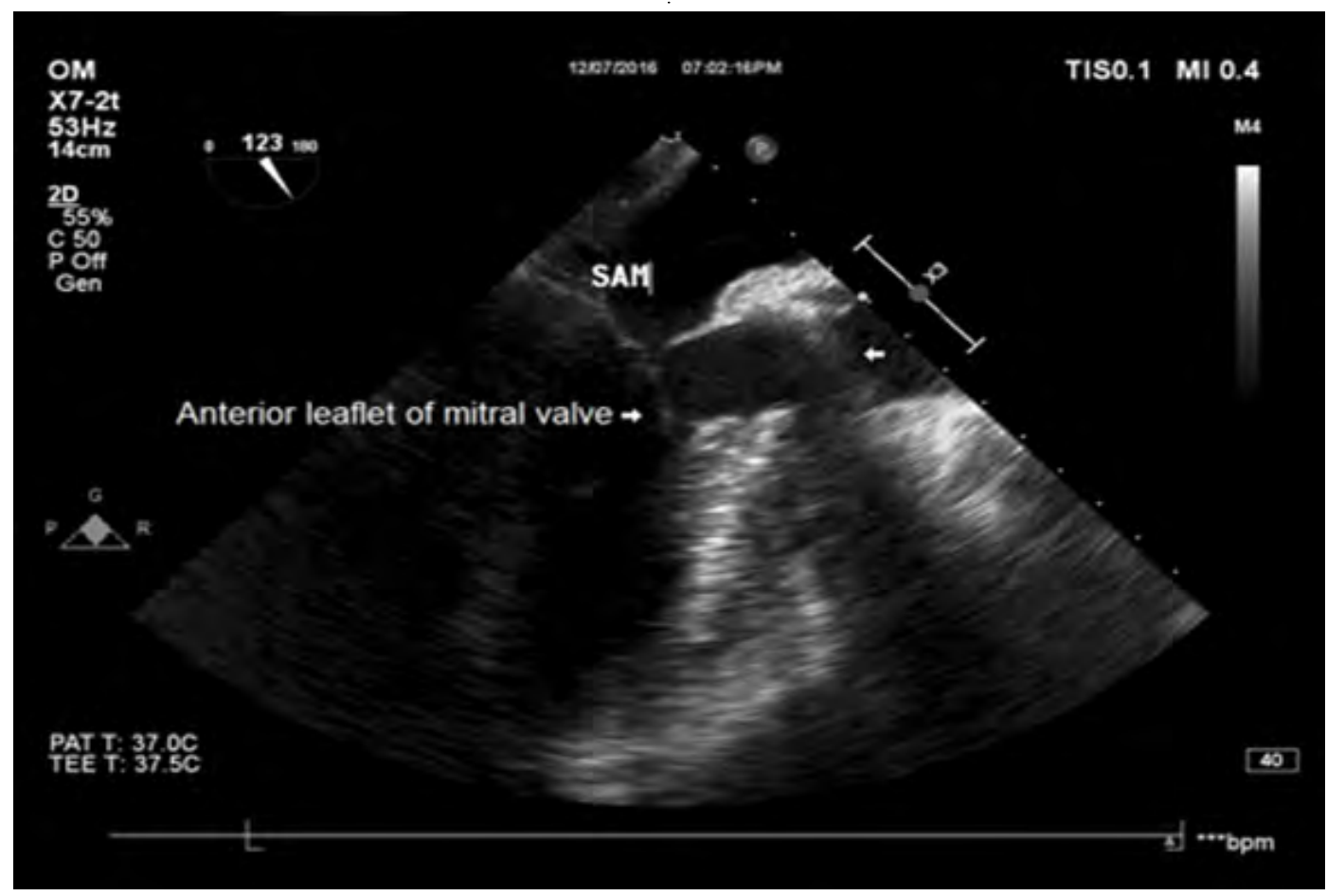

Figure 1: Transesophageal echo still-image showing the anterior mitral leaflet touching the intraventricular septum during systole, diagnosed as systolic anterior motion of the mitral valve.

\section{Discussion:}

We believe that SAM could be strongly attributed to the physiologic changes associated with AVR, itself, as evidenced by our care report. We present a patient with aortic valve stenosis with no history of hypertrophic cardiomyopathy who developed hemodynamically significant SAM after AVR during weaning from bypass despite no inotropic support. The etiology of SAM has been attributed to different factors, of them one being the Venturi effect, which is the sucking effect on the valve leaflet due to the reduction in fluid pressure that results when fluid flows through a constricted outlet. Alternatively, SAM can also be caused by more anterior and inward location of the papillary muscles which alter the chordal tension resulting in a push of the leaflets towards the ventricular septum at the beginning of the systole (4). Although SAM is well-reported in cases of hypertrophic cardiomyopathy, hypertensive heart disease, acute myocardial infarction, and post-operatively after mitral valve repair (4), there are fewer cases in literature that describe SAM in association with AVR procedures (5-7).

SAM following AVR may be an under-recognized cause of hemodynamic instability in the perioperative period with some instances of hemodynamically significant SAM occurring days to months after the initial procedure. This highlights the importance of pre-operative and intra-operative echocardiographic assessment in these patients and continued high index of suspicion intraoperatively during the weaning process. Our treatment approach was non-surgical and aimed towards fluid loading and beta blockade to increase end-diastolic LV dimensions and to decrease contractility, while supporting the patient on CPB. Though fluid loading, increasing systemic vascular resistance, and decreasing contractility are accepted management for LVOT obstruction, our case report highlights the importance of identifying the underreported phenomenon, itself. Xu et al conducted a case series with 48 patients who underwent valve replacement for severe aortic stenosis and observed that patients who developed SAM were ones with increased blood flow velocities of the left ventricular outflow and high intracavitary gradients (8). Patients particularly at risk are those with concentric left ventricular hypertrophy or relatively narrow LVOT which may be worsened by reduction in afterload from perioperative hypovolemia or vasodilation $(6,8)$. According to Sukernik et al. (5) patients with aortic insufficiency have dilated left ventricles and wider LVOT, placing them at a lower risk of developing systolic anterior wall motion of the mitral valve. Bartunek et al. (9) studied 100 patients in the largest prospective study of AVR for aortic stenosis, and found LVOT gradients in $14 \%$ of their patients. According to their study the mechanism was a combination of muscular cavity and SAM, and none of their patients 
required surgical treatment.

SAM should be considered when patients exhibit hemodynamic instability after AVR which does not respond to or worsens after usual supportive measures. When SAM is observed initially, it is important to consider that the etiology may be physiological, and a non-surgical treatment approach using fluid resuscitation and beta blockade may be advisable. It should also be kept in mind that offloading with vasodilators, or intra-aortic balloon pump (10) may worsen the hemodynamic situation.

\section{References:}

1.Routledge T, Nashef S. Severe mitral systolic anterior motion complicating aortic valve replacement. Interactive Cardiovascular and Thoracic Surgery. July 2005 486-487

2.Cutrone F, Coyle J, Novoa R, Stewart R, Currie P. Severe Dynamic Left Ventricular Outflow Tract Obstruction Following Aortic Valve Replacement Diagnosed by Intraoperative Echocardiography. Anesthesiology 1990:72:53-566.

3.Kerut EK, Hanawalt C, Dearstine M, Frank R, Everson, C. Mitral Systolic Anterior Motion (SAM) with Dynamic Left Ventricular Outflow Obstruction Following Aortic Valve Replacement. Echocardiography. J

4.Walker CM, Reddy GP, Mohammed TH, Chung JH. Systolic Anterior Motion of the Mitral Valve. J Thoracic Imaging. July 2014; volume 27, number 4
5.Sukernik, Mikhail R. et al. Systolic Anterior Motion of the Mitral Valve After Aortic Valve Replacement for Aortic Insufficiency. Journal of Cardiothoracic and Vascular Anesthesia, Volume 21, Issue 4, $574-576$

6.Kasahara H1, Inoue Y, Suzuki S, Hayashi I. Pseudo systolic anterior motion: potential for severe mitral regurgitation after aortic valve replacement. Ann Thorac Cardiovasc Surg. 2014;20(4):32931. Epub 2012 Dec 26

7.Dohi M1, Doi K, Okawa K, Yamanami M, Yaku H. Delayed-onset systolic anterior motion of the mitral valve after aortic valve replacement for severe aortic stenosis. Int Heart J. 2013;54(5):292-6

8.Xu J1, Wen J, Shu L, Liu C, Zhang J, Zhao W. Mechanism and correlated factors of SAM phenomenon after aortic valve replacement. J Huazhong Univ Sci Technolog Med Sci. 2007 Feb;27(1):72-4.

9.Bartunek J, Sys SU, Rodrigues AC, van Schuerbeeck E, Mortier L, deBruyne B. Abnormal systolic intraventricular flow velocities after valve replacement for aortic stenosis. Mechanisms, predictive factors, and prognostic significance. Circulation 1996;93:712-719.

10.Morewood GH, Weiss SJ. Intra-aortic balloon pump associated with dynamic left ventricular outflow tract obstruction after valve replacement for aortic stenosis. J Am Soc Echocardiography 2000;13:229-231. 